\title{
CONTEXT AWARE ROUTING OF ENTERPRISE USER COMMUNICATIONS
}

\author{
Munmun De Choudhury Hari Sundaram \\ Arts Media Engineering, \\ Arizona State University, Tempe, AZ 85281 \\ Ajita John \\ Dorée Duncan Seligmann \\ Avaya Research \\ Email: \{munmun.dechoudhury, hari.sundaram\}@asu.edu, \{ajita, doree\}@avaya.com
}

\begin{abstract}
This paper develops a context aware framework to address the diverse communication needs of a modern enterprise. Such enterprises are characterized by workers in different locations, subject to different policies, using different communication devices, and having varying degrees of skill sets. This diversity poses challenges in finding the most effective human worker (agent) for tasks like fielding a customer request, helping another agent with additional expert knowledge, or more generally help complete a task like a supply chain exception. We focus on the problem of routing communications to the most effective agent using a spectrum of contextual knowledge: availability, media type, activity, expertise, and location. We determine an optimal 'request-to-agent' routing based on several metrics of effectiveness depending on the communication context. The optimal agent is selected to communicate on a specific media who minimizes the expected duration of interaction while maximizes the probability of successful call completion. Based on our model we have conducted simulations involving context aware and noncontext aware routing scenarios. The results indicate that the context aware routing outperforms other conventional requestrouting techniques. The work presented here can impact routing algorithms, as well as address problems related to enterprise staffing and temporal variation of context for the agent.
\end{abstract}

\section{INTRODUCTION}

Enterprise events in business processes such as a customer contacting customer service, the deadline for a task, or an application parameter crossing a threshold may trigger the need for a group of people to collaborate. In a modern, virtual, and global enterprise, such collaborations are often achieved through communication between people using one or more of the multitude of available media such as voice, video, instant messaging (IM), email or face-to-face. In contrast to user-initiated collaborations, communications middleware such as Hermes [5] use contextual reasoning to automate the otherwise manual steps and decisions selecting who, how, and when to connect users for these collaborations. Such automation attempts to reduce latency in enterprise decisions and provide tractability for communications.

The diversity and intricacies of large modern enterprises present many challenges to the contextual reasoning in middleware. Enterprises may have distributed locations across the globe; their workers may use different communication technologies, be subject to different policies, work in different locations that are conducive to different extents to different media, multi-task, be involved in different kinds of activities, have varying expertise, and work different hours as part of a global workforce. The challenge to automating communications is to capture the diversity and intricacies in a framework that allows efficient routing of communication requests to the most appropriate person on the most appropriate communication media.

In this paper, we focus on routing communications to the most effective (human) agent using a spectrum of contextual knowledge such as availability, media type, activity, expertise, and location. By agent we mean an extension of a traditional contact center agent to be any member of the Enterprise who can be called upon to handle a variety of tasks from fielding a customer request, helping another agent with additional expert knowledge, or more generally help complete a task like a supply chain exception.

Prior work [1] on context aware computing has focused on the collection, dissemination, and processing of context information independent of the application. In [4] the authors present several principles and methodologies for integrating models of attention into human-computer interaction and communication. They discuss methods for inferring the cost of interrupting users based on multiple streams of events including information generated by interactions with computing devices, visual and acoustical analyses, and data drawn from online calendars. They also present methods for learning models from data which is used to compute the expected cost of interruption for a user. However we note that context is a dynamic construct [2,7]; a fact is contextual due to its relationship to a larger set of interrelated facts involved in the application In this paper, we attempt to model context dynamics and deploy it for determining the appropriate agent in an enterprise.

The key contributions of this paper are to: (a) Develop a Request Model to represent incoming requests. (b) Develop an Agent Model. (c) Develop a Request-Agent Matching to select the optimal agent. We use a Bayesian belief network representation to estimate the key decision making parameters - probability of success, expected duration and media usage. The optimal agent is one who maximizes the probability of success while minimizing the expected duration of interaction. (d) Finally we show extensive simulation results by comparing our model against baseline agent selection approaches. Our model is designed to learn data from the contextual variables of the request/agent model. However, the contact centers of today do not capture these contextual parameters. This has motivated us to test and validate our model through simulated data that attempt to emulate real time behavior. Our results show that our optimal context aware matching approach outperforms baseline approach on metrics such as average queue length, expected duration and probability of success.

The rest of this paper is organized as follows. In the next section we present an example application scenario. In sections 3 and 4, we present the request and the agent model respectively. In section 5, we present the simulation methodology and in section 6 , we present the experimental results. We conclude by summarizing our work and presenting our conclusions.

\section{AN EXAMPLE APPLICATION SCENARIO}

We will now describe an enterprise communication scenario to help highlight the key motivations for the work presented in this paper. This scenario has been drawn from a case study on a large geographically distributed retail store. It illustrates how agents that vary in their job function can be drawn into a communication workflow to automate a complex process and how knowledge 
about contextual factors can facilitate a fast turnaround time for enterprise processes.

The scenario revolves around a customer contacting the customer service department of a retail store. Scenarios that evolve similarly have been observed in other domains such as healthcare. A customer contacts the customer service of a retail store with a question about an appliance. The request is handled by an automated communication application which can be modeled as a workflow that sequences a list of communication steps:

(i) The customer is prompted to provide information about the domain of interest (i.e. appliances). The customer is then connected to a regular service agent who works at a fixed location and has access to various media. This agent determines through a conversation with the customer that she needs expert advice on the specific item that the customer is considering for purchase. The agent inputs this information to the workflow handling the request.

(ii) The workflow advances the request to generate a "skills" request to be fielded by an expert on that item. There is a pool of experts who are qualified to handle this request. However, they do not work regular hours and they don't necessarily work at fixed locations. The workflow determines that the best agent to handle the request is an at-home expert who is available and routes the request to that expert.

(iii)After the customer speaks to the expert, she is asked by the system about her decision to purchase the item. She responds that she wants to buy item $\mathrm{X}$ at a local store. The workflow generates a "check-item-in-stock" request to an on-the-floor agent at the store local to the customer. The request is routed to a mobile agent in the stock room who confirms to the customer that the specific item is available.

(iv)The workflow now generates a "place-an-order" request to a sales agent who helps the customer to place the order.

(v) The workflow advances to generate a "get-ready-for-pickup" request to an agent who gets the item ready for pickup by the customer.

There are several key points to note about the above scenario. Agents have different job functions. Some agents perform tasks that are highly communicative in nature (E.g. the sales agent and the regular agent) while others perform tasks with little communication (E.g. the pickup agent in step (v)). At each step, while there is a pool of agents available for selection, not all agents may be available or working at the time. Expertise areas and levels vary from agent to agent. Different agents have access to different types of media. For example, a regular agent in step (i) may have access to voice, video and IM. However, a mobile agent in step (iii) may have access to only a voice medium on a headset. Each agent has a unique capacity limit on each media type (e.g. a maximum of three IM chats, say). Additionally, the location of the mobile agent in step (iii) may determine the conduciveness of the environment to a request on a particular medium. For example, a noisy cafeteria is not conducive to a voice call, but may be fine for an IM chat. An agent's activity is also an important factor to consider when selecting an agent for a request. For example, if the agent is walking, the agent may prefer a voice session over an IM session. However, if the agent is talking to a customer, a text message could be a better option.

The values for parameters such as expertise, location and/or the nature of the current surrounding environment, activity, media/communication access and their current device status and hours of operation for an agent determine his/her effectiveness in handling a request. Some parameters like the hours of operation may act as a simple filter for agent selection. Others are more complex because the effectiveness of an agent for a particular request may depend on one or more of these parameters. For example, some agents may be more effective on voice whereas others may be more effective on IM. While there are several parameters that are relevant to enterprise processes, we consider only those parameters that are relevant to communication routing based on the scenario offered by the case study. Additionally, not all parameters may be relevant for all types of agents. For example, the location parameter may not be relevant for an agent with a fixed location. Also, it may not be possible to monitor all parameters for all agents. In such cases, distributions may have to be assumed for each agent type.

\section{THE REQUEST MODEL}

A request refers to a communication that has to be routed to an agent. In the request model, we consider semantic category (domain) and priority as the key contextual variables (Table 1).

Table 1: Attributes of the request model

\begin{tabular}{ccc}
\hline Type & Variable & Explanation \\
\hline Semantic Category & $C_{r}$ & $C_{r}$ is the semantic category \\
of request $i$.
\end{tabular}

Incoming requests are classified into requests in several different semantic categories. When there are many requests arriving concurrently, the priority of a request is decided on the basis of the semantic category it belongs to and by customer importance.

\section{THE AGENT MODEL}

In our work, an agent refers to an extension of a traditional contact center agent. She can be any member of the Enterprise who can be called upon to handle a variety of tasks from fielding a customer request, helping another agent with additional expert knowledge, or more generally help complete a task like a supply chain exception. In this section we have identified the key agent attributes which characterize the agents. These attributes are not exhaustive, but are consistent with the domains of our interest. Table 2 describes the agent model variables.

The selection of an agent is dependent upon his availability for work (office hours, outlook calendars). An agent can be involved in several different activities (like walking on the road or talking) at different locations (like home or office), at any point in time. There can be several media types available for communication to an agent (like voice or instant messaging) that determine the current context. Knowledge of the media usage is important since for a maximum capacity $c_{j}^{k}$ on the media type $M_{k}$, we can determine how close the agent is to his full capacity. This can help us determine how effectively he can handle a new communication task. Additionally, the agent should have the requisite expertise in the particular semantic category of the request.

\section{AGENT SELECTION FRAMEWORK}

In this section, we identify certain optimization parameters (Table 3 ) and their dependence on the contextual variables in the request/agent models. We also define an optimized matching 
metric for the successful routing of a request to determine the agent.

\subsection{Optimization Parameters}

The goal of every service-oriented enterprise is to render the best possible service to its customers through an optimal handling of a request. Factors related to quality service are: (1) Was the call handled adequately by the agent? (2) Did the agent complete the call to the customer's satisfaction in reasonable amount of time? (3) How can we capture the aspects of call success that are related to the conduciveness of the medium and which are associated with the media dynamics over different points of time and environment? Absence of a way to address these issues often would give rise to dropped calls, customer not being satisfied, customer rarely calling back again due to long queue wait times, unacceptably long service times etc.

We have described a set of optimization parameters catering to these issues (Table 3) through two example cases. These parameters are based on the request and the agent context and we refer to them as a mechanism for an optimal 'request-agent' matching.

Table 2: Agent model attributes

\begin{tabular}{|c|c|c|}
\hline Type & Variable & Explanation \\
\hline $\begin{array}{l}\text { Availability (Hours of } \\
\text { Operation) }\end{array}$ & Boolean & $\begin{array}{c}\text { We use availability as a } \\
\text { predicate }\end{array}$ \\
\hline Location & $L_{j}$ & $\begin{array}{c}L_{j} \text { is the location of agent } \\
j .\end{array}$ \\
\hline Activity & $A_{j}$ & $\begin{array}{c}A_{j} \text { is the activity of agent } \\
\mathrm{j} .\end{array}$ \\
\hline Media Type & $M_{k}$ & $M_{k}$ is the $k^{\text {th }}$ media type \\
\hline $\begin{array}{l}\text { Media Usage and } \\
\text { Capacity }\end{array}$ & $U_{j}^{k}, c_{j}^{k}$ & $\begin{array}{l}\text { Media Usage } U_{j k} \text { refers } \\
\text { to the number of media } \\
\text { channels of type } M_{k} \text { that } \\
\text { an agent } j \text { is using at this } \\
\text { moment. } c_{j}^{k} \text { is the } \\
\text { maximum number of } \\
\text { requests she can attend. }\end{array}$ \\
\hline Expertise & $E_{j}^{r}$ & $\begin{array}{l}E_{j}^{r} \text { is the expertise of } \\
\text { agent } j \text { on the request } \\
\text { semantic category } C_{r} \text {. }\end{array}$ \\
\hline
\end{tabular}

Consider an agent with high expertise (with respect to the request) and who is currently handling two instant messaging requests. She is more likely to succeed and complete the request in less time than an agent with moderate expertise (with respect to the same request), handling more requests and using one voice channel and two instant messaging communications. The first agent is an expert with a lower cognitive load and is hence more likely to succeed. The probability of success and estimate of duration depends upon the current media capacity, the current Media Usage $U_{j}^{k}$ and the expertise $E_{j}^{r}$ of the agent.

Table 3: Request-Agent Matching attributes
Type
Variable
Explanation

\begin{tabular}{ccc}
\hline $\begin{array}{c}\text { Probability of Media } \\
\text { Usability }\end{array}$ & $u_{j}^{k}$ & $\begin{array}{c}u_{j}^{k} \text { is the media usability of } \\
\text { agent } j \text { on media type } M_{k} .\end{array}$ \\
\hline Probability of Success & $s_{j}$ & $\begin{array}{c}s_{j} \text { is the probability of } \\
\text { success of agent } j .\end{array}$ \\
\hline $\begin{array}{c}\text { Estimate of Duration of } \\
\text { Interaction }\end{array}$ & $d_{j}$ & $d_{j}$ is the estimate of duration \\
of agent $j$.
\end{tabular}

In a second example consider an agent on the floor of a retail store with voice and instant messaging capabilities. The location and activity of the agent suggests that she is in a noisy environment. Therefore, IM is a better way to route a request to her than voice because the media usability (voice) is not well matched to the environment. Hence it is important to know the media usability before routing a communication. The media usability depends upon the location, activity of the agent and the media type.

\subsection{Bayesian estimation}

In this section, we construct three Bayesian networks for determining probability of success $s_{j}$, estimating duration of interaction $d_{j}$ and probability of the media usability $u_{j}^{k}$ for a given media type $M_{k}$. The probability of success $s_{j}$ is as follows:

$P\left(s_{j} \mid E_{j}^{r}, M_{k}, U_{j}^{k}\right)=\frac{P\left(s_{j}, E_{j}^{r}, M_{k} U_{j}^{k}\right)}{P\left(E_{j}^{r}, M_{k} U_{j}^{k}\right)}$

The duration of interaction $d_{j}$ depends upon three factors: the domain expertise, media usage and the media type. It is conditionally independent of $\mathrm{P}\left(s_{j}\right)$ given these three variables. The estimate of duration $d_{j}$ :

$$
\begin{aligned}
P\left(d_{j} \mid E_{j}^{r}, M_{k} U_{j}^{k}\right) & =\frac{P\left(d_{j}, E_{j}^{r}, M_{k} U_{j}^{k}\right)}{P\left(E_{j}^{r}, M_{k} U_{j}^{k}\right)}, \\
E\left(d_{j}\right) & =d_{j} * P\left(d_{j}\right)
\end{aligned}
$$

We estimate the media usability $u_{j}^{k}$ of a particular media type $M_{k}$ with an agent $j$ as follows:

$$
P\left(u_{j}^{k} \mid L_{j}, A_{j,} M_{k}\right)=\frac{P\left(u_{j}^{k}, L_{j}, A_{j}, M_{k}\right)}{P\left(L_{j}, A_{j}, M_{k}\right)}
$$

These three estimates play a critical role in determining the optimal agent.

\subsection{The Metric}

In this section, we define a metric for selection of the optimal agent. The optimal agent will be the one who has maximum success and minimum expected duration of interaction. This is computed as follows:

$$
z_{j} \longleftarrow\left\{\max _{M_{k}}\left(\frac{P\left(s_{j} \mid E_{j}^{r}, M_{k}, U_{j}^{k}\right)}{E\left(d_{j} \mid E_{j}^{r}, M_{k}, U_{j}^{k}\right)}\right)\right\}
$$

This estimate needs to be filtered by the suitability of the media type for the interaction. Hence the optimality criterion is modified as follows:

$z_{j} \longleftarrow\left\{\max _{M_{k}}\left[\left(\frac{P\left(s_{j} \mid E_{j}^{r}, M_{k}, U_{j}^{k}\right)}{E\left(d_{j} \mid E_{j}^{r}, M_{k}, U_{j}^{k}\right)}\right) * P\left(u_{j}^{k} \mid L_{j}, A_{j}, M_{k}\right)\right]\right\}<5>$ 
In the next section we describe our simulation methodology. As mentioned earlier, simulation is necessary to validate our ideas, as real-world enterprise datasets do not capture the rich contextual information that are needed for optimal agent selection.

\section{SIMULATION METHODOLOGY}

In this section we discuss the methodology adopted for the simulation. The main goals of our simulation are:

- To identify a set of agent categories (mutually exclusive) and to examine if they are exhaustive, useful, realistic and appropriate to the problem under consideration. Members of the same category are assumed to exhibit more or less similar kind of behavior.

- To identify 'Critical Attributes' for each agent category. $A$ critical attribute is a contextual variable whose distribution is statistically different from the average distribution of the rest of the whole population (e.g. by computing the relative entropy on the distributions).

- Construct empirical distributions for the contextual variables discussed in previous section.

- Validate the matching criteria.

In a real-world scenario, every agent has a very different context. Motivated by our example scenarios (ref. section 2) we define six mutually exclusive agent classes. We then determine the critical attributes corresponding to each category.

- Mobile Agents: Location, Media Type, Hours of Operation and Capacity are critical attributes for this category. Mobile Agents frequently change locations and they have fixed hours of operation. While working they would use several communication technologies to serve customers and would have a fixed capacity.

- Regular Agents: Media Type, Hours of Operation and Capacity are critical attributes for this category. They work mostly at the office (on-site) and have fixed office hours. So Location is not a critical attribute. They have access to a wide range of communication devices and have a fixed capacity.

- Stay-at-Home Agents: Media Type, Hours of Operation and Capacity are critical attributes for this category. They work mostly from home and have fixed office hours. Location is not a critical attribute. They can use several technology devices to handle requests and have a defined capacity.

- High Communication Agents: Media Type and Capacity are critical attributes for this category. We conjecture that Location and Hours of Operation are not critical for them.

- Low Communication Agents: Media Type, Capacity, Hours of Operation and Location are the critical attributes because they characterize their low degree of communication.

- Reserve Agents: Media Type and Location are critical attributes for this category because they are likely to mobile and can have several media types available. Since they are utilized when needed their hours of operation are not fixed.

Table 4: Contextual attributes and values

\begin{tabular}{cc}
\hline $\begin{array}{c}\text { Contextual } \\
\text { Attributes }\end{array}$ & Values \\
\hline Location & $\begin{array}{l}\text { Office, Home, Mall, Own Office, Conference } \\
\text { Room, Home Office, Retail Floor, Customer }\end{array}$ \\
\hline
\end{tabular}

\begin{tabular}{cc}
\hline & Support, Hotel \\
\hline Activity & $\begin{array}{c}\text { Interacting, Talking, At a Meeting, Sleeping, } \\
\text { Traveling, Walking, Jogging }\end{array}$ \\
\hline Media Type & e-mail, IM, voice, video, face-to-face interaction \\
\hline Capacity & $0,1,2,3$ requests \\
\hline
\end{tabular}

We have represented the contextual variables with the help of several empirical distributions for the generation of simulation data. We have assumed a set of exhaustive values of the variables which an agent might assume in any real life scenario in Table 4.

\subsection{Simulation Procedure}

We assume that we have agents distributed in the following way as per the choice of the agent categories discussed in last section and their probable staffing in a typical contact center: $20 \%$ (mobile), 30\% (office), 12\% (stay at home), 25\% (high comm.) $3 \%$ (low comm.) and 10\% (back up). We assume a Poisson arrival of the requests [3], since a Poisson distribution expresses the probability of a number of events (request arrival) occurring in a fixed period of time if these events occur with a known average rate (chosen as per typical statistical arrival measures in contact centers), and are independent of the time since the last event (arrival of request $i$ is generally independent of a prior request $j$ ). Let us assume that at time $t, N$ requests arrive corresponding to different domains. We now decide on the priorities of the requests from a distribution (exponential) of priorities over different request semantic categories $C_{r}$ discussed in Section 3.

A unique agent profile is also constructed which would describe the agent's Hours of Operation distribution. The distributions are chosen in a way which reflects real life employee behavior in enterprises. For example, an agent is more likely to be working late hours earlier in the week (Monday, Tuesday) than on Friday. Few of the Regular or Stay-at-Home Agents will be working in the weekends. Again the hours of operation of the High Communication Agents will be more than the Reserve Agents. On the basis of such distributions, we determine which agents are available at that instant; and, if available, if the agent has expertise with respect to the domain of the request. We check the Media Usage of the agent as that time instant. Only those agents who are below their maximum capacity are considered. The short-listed agents are the potential agents.

In a real-world scenario, contact center data (comprising of the context aware parameters) would be available that could be learnt by a Bayesian network, which can estimate the duration. However, for the purposes of the simulation, we compute the expected duration as follows. The expected duration for each agent is assumed to be related to three elements: expertise on the semantic category of the request, media type and current media usage. For the purpose of the simulation, we have assumed that the parameters are conditionally independent given duration. Then, duration is varied exponentially over expertise, the set of media types and on the current media usage. The parameters for the exponential distributions are determined empirically.

The optimal agent who can yield maximum success in least duration over a Media Type is determined using equation $<5>$. The simulation is started by assuming a uniform prior on the probability of success - this estimate is revised as part of the simulation. A coin (Bernoulli variable with parameter equal to the probability of success) is tossed to decide if the agent succeeded 
in servicing the request. If he did, then the probability of success is updated for the corresponding values of media type, media usage and expertise. If all of the agents are full to their maximum capacity, the request goes to a request queue.

\section{EXPERIMENTAL RESULTS}

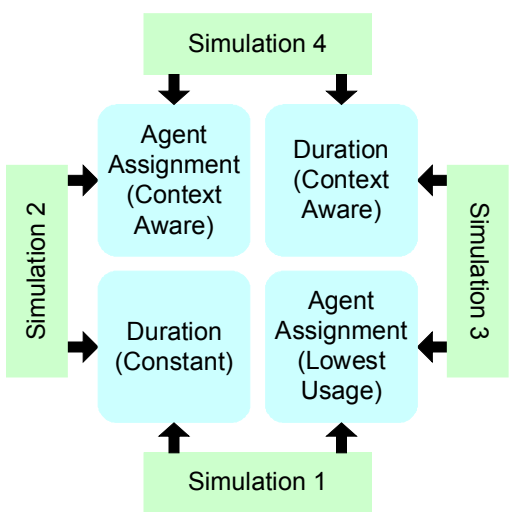

Figure 1: We use four simulation scenarios for comparing our approach with baseline approaches.

We considered four different simulation scenarios (Figure 1) in our experiments to compare our context aware simulation to three other baseline simulation scenarios.

\subsection{Simulation Scenarios}

Three of the simulations (1-3) are either non-context aware in terms of agent assignment and estimated duration of interaction, or they involve only one of these two parameters to be contextaware. The notion is to deploy several different (traditional) metrics of request-agent matching like, request routing based on 'lowest media usage agent' or routing based on a fixed (average) estimated duration of interaction [6]. The goal, thereafter, is to consider different parameters of comparison (the behavior of request queue length over time, average probability of success and average estimate of duration) and verify and validate that the context-aware routing model performs best among the four. Request queue length is a good metric because a good model for request routing will minimize the request service queue length. Also it will yield better average success and better duration.

In Simulation 1, for an optimal selection of agent, we do an agent assignment based on lowest media usage. The estimate of duration is assumed to be constant at 10 minutes (say). In Simulation 2, we do an agent assignment based on the context models but the estimate of duration is assumed to be constant. In Simulation 3, the agent assignment is based on lowest media usage, but we use a context aware estimate of duration as discussed in Section 5. Finally, in Simulation 4, both the parameters are chosen to be context-aware as per the model discussed in Section 5.

We considered a Poisson request arrival rate of $\lambda=25$ requests/min. The behaviors of the request queue length for 60 agents over time for four different simulations are shown in Figure 2.

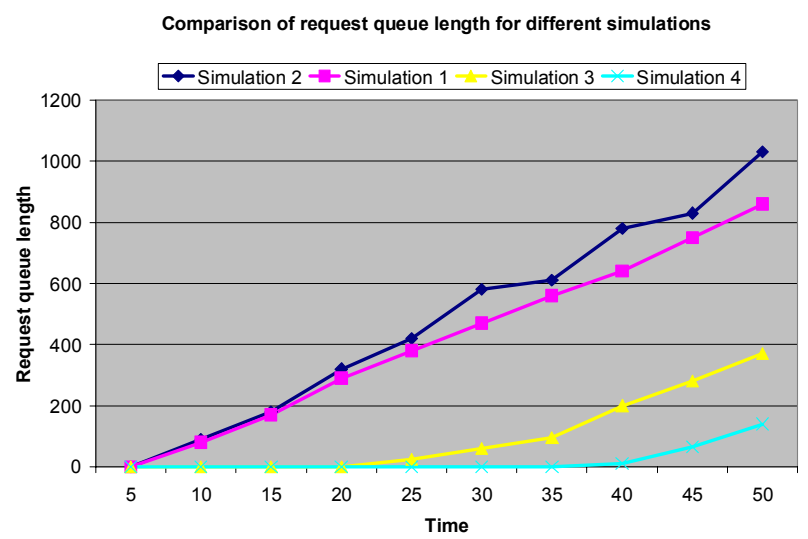

Figure 2: Variations in request queue length for the four simulation scenarios. Note that the expected request queue length is shortest in the context aware case.

\subsection{Validation of Results}

We find that the request queue for the context aware case starts to increase a much later stage (at $t=41$ ) compared to other matching schemes (rate of change of queue length over time). Figure 3 and Figure 4 show the plots of the average probability of success and average estimate of duration of interaction for all four simulations for six different sets of agents $(20,50,75,150,200$ and 250).

It is easily observable that for different sets of agents (differing in expertise, availability, capacity and media usage) as well as different arrival rates of requests, Simulation 4 yields maximum probability of success for a particular request routing, yields minimum estimated duration of interaction, and attempts to 'match' as many requests as possible without letting the request queue grow exponentially over time, as with the other three scenarios. This implies that our chosen metric for context-aware request-agent matching (Section 5) is a more satisfactory measure than the traditional metrics chosen in the other three scenarios which are more usually deployed in today's contact centers. The result is a minimal waiting time for customers (request queue length) and a promising degree of success (in handling of the request) within a reasonable amount of time as well.

These findings validate our context aware model of routing of requests in an enterprise scenario.

\section{CONCLUSIONS AND CONTRIBUTIONS}

In this paper, we have developed a model for decision making for communication routing in a modern day contact center. We developed (a) agent and request models, (b) Bayesian network based frameworks for determining the optimal agent. The optimal agent is one who maximizes the ratio of the probability of success to the expected duration for a usable media type, and (c) performed simulation over empirical distributions of attribute values. Simulations indicate that the context aware model outperforms other models which validates the fact that the use of context in a modern day enterprise as a call center is an ideal means to automate communication and for effective decision making in terms of communication routing. 


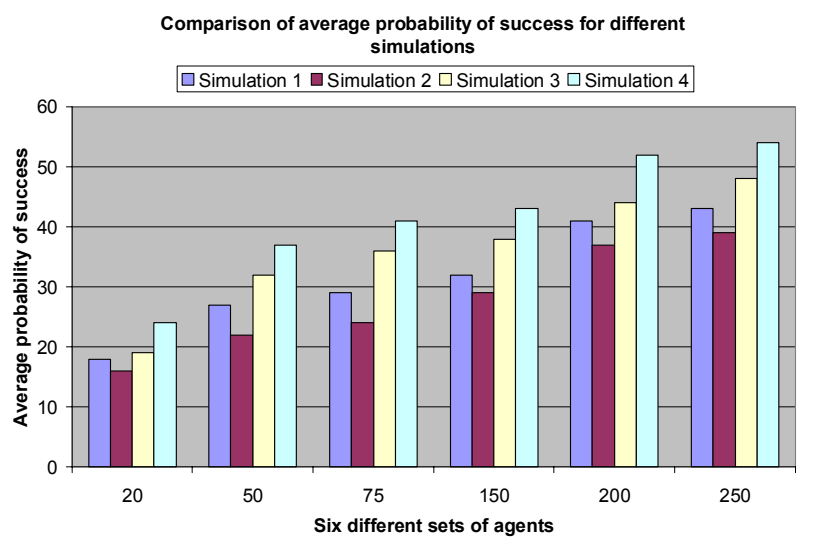

Figure 3: Average probability of success. The context aware case maximizes the probability of success.

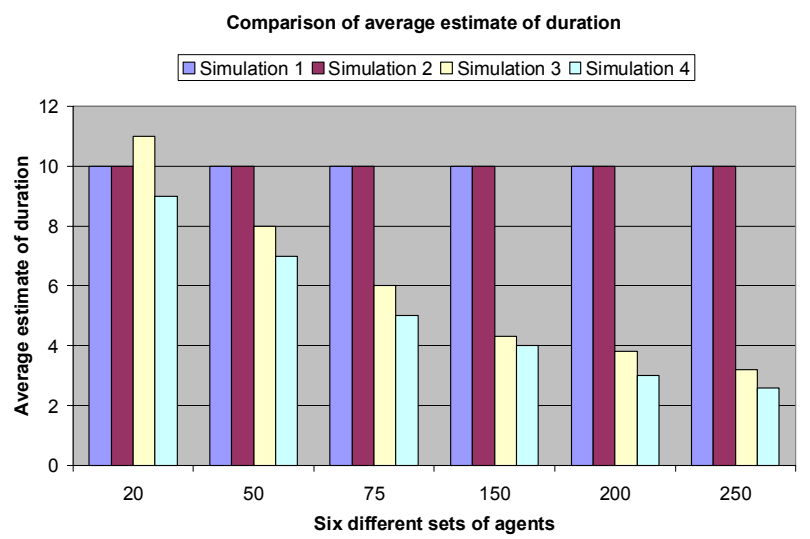

Figure 4: Average duration of interaction. This is minimum for the context aware routing scenario.

We seek to extend this research in future in several ways - (a) Contextual correlation: In the analysis in the paper, we assumed independence of different contextual attributes. For example, in a real world scenario it is likely that a particular media type for a specific agent may preclude the simultaneous use of another media type. In general the correlation across the contextual attributes can be modeled at the agent level; (b) Enterprise staffing: Given an enterprise call scenario (request category, time of call etc.) how to determine the optimal staffing distribution that minimizes cost and maximizes probability of success; and (c) Agent optimization: Agents may have contexts that change over time (e.g. agent is recuperating at home, emergency travel etc.) how to adapt the call routing to the agent so that she can be maximally effective.
Nevertheless, this paper gives a broad overview on the depth of problems faced in modern contact centers in routing of requests to agents as well as suggests a context-aware solution to overcome the problem. This can also impact the conventional staffing criteria in contact centers as well as can address better customer/client relationships with the enterprise with respect to fewer dropped customer calls, better quality of customer service, while these two remain big challenges in the service industry of today.

\section{REFERENCES}

[1] A. K. DeY (2001). Understanding and Using Context. Personal and Ubiquitous Computing Journal. 5: 4-7.

[2] P. Dourish (2004). What we talk about when we talk about context. Personal and Ubiquitous Computing. 8: 19-30.

[3] J. H. Haipeng Shen (2005). Analysis of Call Center Arrival Data Using Singular Value Decomposition. Applied Stochastic Models in Business and Industry 21(251-263).

[4] E. Horvitz, C. M. Kadie, T. Paek, et al. (2003). Models of Attention in Computing and Communications: From Principles to Applications. Communications of the ACM. 46: pp. 52-59.

[5] A. John, R. Klemm, A. Mani, et al. (2006). Hermes: A Platform for Context-Aware Enterprise Communication, Proceedings of the Fourth Annual IEEE International Conference on Pervasive Computing and Communications Workshops, 62-67,

[6] N. G. Lawrence D. Brown, Avishai Mandelbaum, Anat Sakov, Haipeng and S. Z. Shen, Linda H. Zhao (2005). Statistical Analysis of a Telephone Call Center: A QueueingScience Perspective. Journal of the American Statistical Association 100: 36-50.

[7] A. Mani and H. Sundaram (2006). Modeling User Context with Applications to Media Retrieval. to appear in Multimedia Systems Journal, Summer 2006. 